\title{
Complete Regular Fuzzy Graphs
}

\section{S. Venu Madhava Sarma, R.Sunil Kumar, T.V. Pradeep Kumar}

\begin{abstract}
In this paper, some properties of complete degree and complete regular fuzzy graphs are discussed. They are illustrated through various examples. It is proved that every fuzzy graph is an induced subgraph of a complete regular fuzzy graph. The procedure described in the proof is illustrated through an example. Also the complete degree of a vertex in fuzzy graphsformed by the operation Union in terms of the complete degree of vertices in the given fuzzy graphs for some particular cases are obtained. Using them, their complete regular property is studied.
\end{abstract}

\section{INTRODUCTION}

Azriel Rosenfeld was introduced Fuzzy graph theory in 1975 [9]. Bhattacharya [1] gave some remarks on fuzzygraphs.Mordeson.J.N. and Peng.C.S.were introduced Some operations on fuzzy graphs [4]. Zadehintroduce a mathematical frame work to describe the phenomena of uncertainty in real life situation has been suggested in 1965[3].

Research on the theoryof fuzzy sets has been witnessing an exponential growth of both mathematics and in its applications. This rangesfrom traditional mathematical subjects like logic topology, algebra, analysis etc. to pattern recognition, information theory, artificial intelligence, operations research, neural networks and planning etc. Mordeson.J.N. and Peng.C.S were defined by the operations of union, join, Cartesian product

and composition on two fuzzy graphs [4]. Nagoorgani.A and Radha. K. was discussed the degree of a vertex in some fuzzygraphs and the Regular property of fuzzy graphs which are obtained from two given fuzzy graphs using the operations union,join, Cartesian product and composition [8].

In this paper we study aboutsome

properties of complete regular fuzzy graphs. First we go through some basic definitions which can be found in [1-13].

Definition 1.1 ([2]).“A fuzzy subset of a set $\mathrm{V}$ is a mapping $\sigma$ from $\mathrm{V}$ to $[0$, 1]. A fuzzy graph $\mathrm{G}$ is a pair of functions $\mathrm{G}:(\sigma, \mu)$ where $\sigma$ is a fuzzy

Revised Manuscript Received on November 11, 2019.

S. Venu Madhava Sarma, Associate Professor of Mathematics, K L E Foundation University, Vaddeswaram Post, Guntur.

R.Sunil Kumar, Associate Professor of Mathematics, K L E Foundation University, Vaddeswaram Post, Guntur

T.V. Pradeep Kumar, Assistant Professor of Mathematics, A N U College of Engineering, Acharya Nagarjuna University, Andhra Pradesh, Guntur subset of a non empty set $\mathrm{V}$ and $\mu$ is a symmetric fuzzy relation on $\sigma$, i.e., $\mu(\mathrm{uv})=\sigma(\mathrm{u}) \wedge \sigma(\mathrm{v})$. The underlying crisp graph ofG : $(\sigma, \mu)$ is denoted by $\mathrm{G} *$ : $(\mathrm{V}, \mathrm{E})$, where $\mathrm{E} \subseteq \mathrm{V} \times \mathrm{V}$

Definition 1.2 ([2]).G0 : $(\sigma 0, \mu 0)$ is a fuzzy sub graph or a partial fuzzy sub graph of $\mathrm{G}:(\sigma, \mu)$ if $\sigma 0 \subseteq \sigma$ and $\mu 0 \subseteq$ $\mu$; that is if $\sigma 0(\mathrm{u}) \leq \sigma(\mathrm{u})$ for every $\mathrm{u}$ $\in \mathrm{V}$ and $\mu 0(\mathrm{uv}) \leq \mu(\mathrm{uv})$ for everyuv $\epsilon$ E.

Definition 1.3 ([2]). G0 : $(\sigma 0, \mu 0)$ is a fuzzy spanning sub graph of $\mathrm{G}:(\sigma, \mu)$ if $\sigma 0=\sigma$ and $\mu 0 \subseteq \mu$; that is if $\sigma 0(\mathrm{u})=\sigma(\mathrm{u})$ for every $\mathrm{u} \in \mathrm{V}$ and $\mu 0($ uv) $\leq \mu(u v)$ for every uve E. Definition 1.4 ([2]). For any fuzzy subset ' $v$ ' of $\mathrm{V}$ such that $v \subseteq \sigma$, the fuzzy sub graph of $\mathrm{G}:(\sigma, \mu)$ induced by $v$ is the maximal fuzzy sub graph of $\mathrm{G}:(\sigma, \mu)$, that has fuzzy vertex set $v$ and it is the fuzzy sub graph $\mathrm{H}$ : $(v, \tau)$ where $\tau(\mathrm{u}, \mathrm{v})=\tau(\mathrm{u}) \wedge \tau(\mathrm{v}) \wedge \mu(\mathrm{u}, \mathrm{v})$ for all $\mathrm{u}, \mathrm{v}$ in $\mathrm{V}$

Definition 1.5 ([7]). Let $\mathrm{G}:(\sigma, \mu)$ be a fuzzy graph on $\mathrm{G} *$ : $(\mathrm{V}, \mathrm{E})$. The degree of $a$ vertex $u$ is

$\mathrm{dG}(\mathrm{u}) \quad=\sum(\mathrm{u} \neq \mathrm{v})$ 洋 $\mathrm{u}(\mathrm{uv})$. The minimum degree of $\mathrm{G}$ is $\delta(\mathrm{G})=\wedge\{\mathrm{dG}(\mathrm{v}), \quad \forall \quad \mathrm{v} \in \mathrm{V}\}$ and the

maximum degree of $\mathrm{G}$ is $\Delta(\mathrm{G})=$ $\mathrm{V}\{\mathrm{dG}(\mathrm{v}), \quad \forall \quad \mathrm{v} \in \mathrm{V}\}$.

Definition 1.6 ([5]). The order and size of a fuzzy graph $G$ are defined by $O(G)$

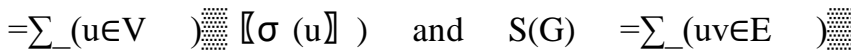
$\llbracket \mu(\mathrm{uv}) . \rrbracket$

Definition 1.7 ([7]). Let $G \quad: \quad(\sigma, \mu)$ be a fuzzy graph on $\mathrm{G}^{*}:(\mathrm{V}, \mathrm{E})$. Then $\sigma$ is a constant function if and only if thefollowing are equivalent:

1. $\mathrm{G}$ is a regular fuzzy graph.

2. G is a Complete regular fuzzy graph".

Note:"Throughout this paper $\mathrm{G}_{1} *:\left(\sigma_{1}, \mu_{1}\right)$ and $\mathrm{G}_{2} *:\left(\sigma_{2}, \mu_{2}\right)$ denote two fuzzy graphs with underlying crisp graphs $\mathrm{G} 1 *$ : $(\mathrm{V} 1, \mathrm{E} 1)$ and $\mathrm{G}_{2} *$ : $\left(\mathrm{V}_{2}, \mathrm{E}_{2}\right)$ with $|\mathrm{Vi}|=\mathrm{pi}, \mathrm{i}=1,2$. Also $\mathrm{dG} * \mathrm{i}$ (ui) denotes the degree of ui in $\mathrm{G} * \mathrm{I}$ ".

Definition 1.8 ([8]). The union of two fuzzy graphs G1 and $\mathrm{G} 2$ is defined as a fuzzy graph $\mathrm{G}=\mathrm{G}_{1} \cup \mathrm{G}_{2}:\left(\sigma_{1} \cup \sigma_{2}, \mu 1 \cup\right.$ $\left.\mu_{2}\right)$ on $\mathrm{G} *(\mathrm{~V}, \mathrm{E})$ where $\mathrm{V}=\mathrm{V}_{1}$ $\mathrm{U} \mathrm{V}_{2}$ and $\mathrm{E}=\mathrm{E}_{1} \cup \mathrm{E}_{2}$ with 


\section{Complete Regular Fuzzy Graphs}

$$
\begin{array}{r}
\left(\sigma_{1} \cup \sigma_{2}\right)(\mathrm{u})=\left\{\begin{array}{ll}
\sigma_{1}(u), & \text { if } u \in V_{1}-V_{2} \\
\sigma_{2}(u), & \text { if } u \in V_{2}-V_{1} \\
\sigma_{1}(u) V \sigma_{2}(u), & \text { if } u \in V_{2} \cap V_{1}
\end{array}\right\} \\
\left(\mu_{1} \cup \mu_{2}\right)(\mathrm{u})=\left\{\begin{array}{ll}
\mu_{1}(e), & \text { if } e \in E_{1}-E_{2} \\
\mu_{2}(u), & \text { if } e \in E_{2}-E_{1} \\
\mu_{1}(e) V \sigma_{2} e, & \text { if } e \in E_{2} \cap E_{1}
\end{array}\right\}
\end{array}
$$

Definition 1.9 ([7]). Let $G:(\sigma, \mu)$ be a fuzzy graph. The degree of a vertex $u$ in $G$ is defined by

$$
\mathrm{d}_{\mathrm{G}}(\mathrm{u})=\sum_{u \neq v} \mu(u v)=\sum_{u v \in E} \mu(u v)
$$

Definition $1.10([8])$.“Let $\mathrm{G}:(\sigma, \mu)$ be a fuzzy graph on $\mathrm{G} *$. The total degree of a vertex $u \in V$ is defined by

$$
\operatorname{td}_{\mathrm{G}}(\mathrm{u})=\sum_{u \neq v} \mu(u v)+\sigma(u)=\mathrm{d}_{\mathrm{G}}(\mathrm{u})+\sigma(u)
$$

If each vertex of $G$ has the same complete degree $k$, then $G$ is said to be a complete regular fuzzy graph of complete degree $\mathrm{k}$ or a $\mathrm{k}$ - completeregular fuzzy graph.

Notation 1.11. The relation $\sigma 1 \leq \mu 2$ means that $\sigma 1(\mathrm{u}) \leq \mu 2(\mathrm{e})$ $\forall \mathrm{u} \in \mathrm{V} 1$ and $\forall \mathrm{e} \in \mathrm{E} 2$ where $\sigma 1$ is a fuzzy subset of V1 and $\mu 2$ is a fuzzy subset of $E 2$.

Lemma 1.12 ([8]). If G1 $:(\sigma 1, \mu 1)$ and $\mathrm{G} 2:(\sigma 2, \mu 2)$ are two fuzzy graphs such that $\sigma 1 \leq \mu 2$ then $\sigma 2 \geq \mu 1$.

Definition 1.13 ([7]Total degree of a vertex). Let $\mathrm{G}:(\sigma, \mu)$ be a fuzzy graph on $\mathrm{G} *$ : $(\mathrm{V}, \mathrm{E})$. The total degree of a vertex $u \in$ $\mathrm{V}$ is defined by

$$
\operatorname{td}_{\mathrm{G}}(\mathrm{u})=\sum_{u \neq v} \mu(\mathrm{uv})+\sigma(\mathrm{u}) \text { '. }
$$

Example 1.14. Consider the following fuzzy graph $G:(\sigma, \mu)$.

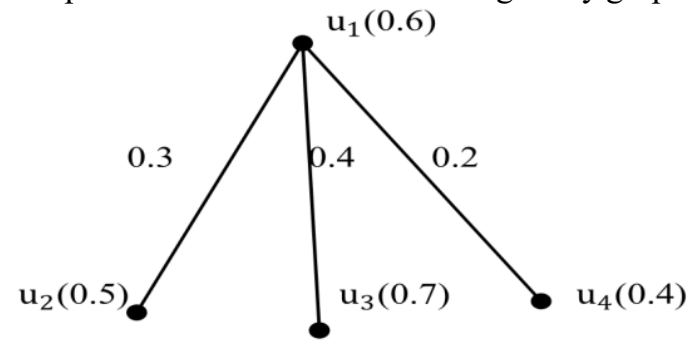

Figure 1.

$\operatorname{tdG}(\mathrm{u} 1)=[\mu(\mathrm{u} 1 \mathrm{u} 2)+\mu(\mathrm{u} 1 \mathrm{u} 3)+\mu(\mathrm{u} 1 \mathrm{u} 4)]+\mathrm{s}(\mathrm{v})=[0.3+0.4+$ $0.2+0.6]=1.5$

Similarly,

$\operatorname{tdG}(\mathrm{u} 2)=0.8 ; \operatorname{tdG}(\mathrm{u} 3)=1.3 ; \mathrm{tdG}(\mathrm{u} 4)=0.6$

2.Complete Regular Fuzzy Graph

" Let $\mathrm{G}:(\sigma, \mu)$ be a fuzzy graph on $\mathrm{G} *:(\mathrm{V}, \mathrm{E})$. If each vertex in Ghassame total degreek,then Gissaid to be a Complete regular fuzzy graph or k- complete regular fuzzy graph".

Example :2.1Consider the following fuzzy graph $G:(\sigma, \mu)$

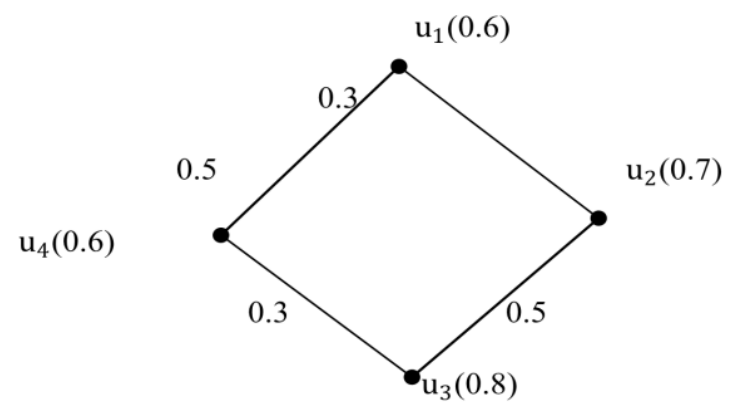

Figure 2.

"The fuzzy graph in Figure 2 is a 1.2- Complete regular fuzzy graph. Also it is a 0.6-regular fuzzy graph".

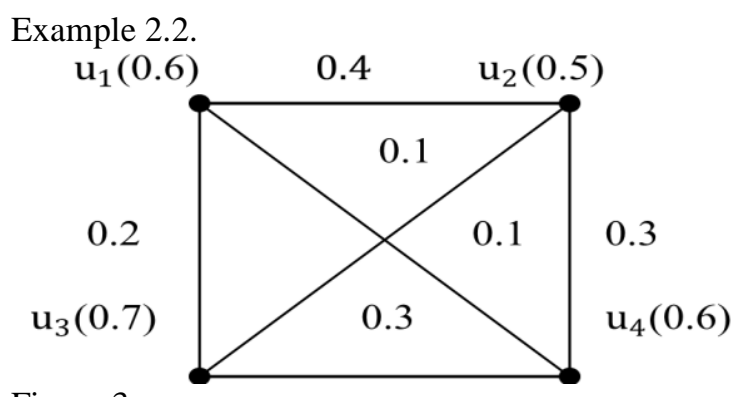

Figure 3.

" The fuzzy graph in Figure 3 is a 1.3- complete regular fuzzy graph. But it is not a regular fuzzy graph".

Example 2.3.

$u_{2}(0.6)$

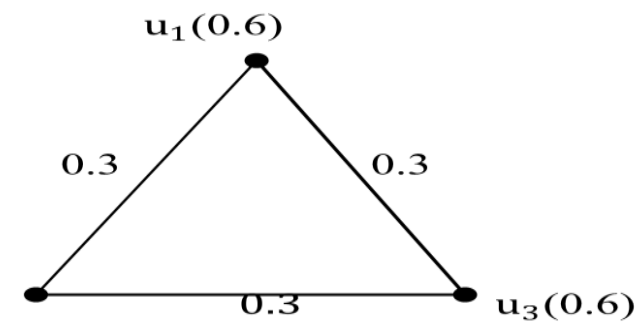

Figure 4.

“ The fuzzy graph in Figure 4 is a 0.8-regular fuzzy graph. But it is not a complete regular fuzzy graph".

Example 2.4.

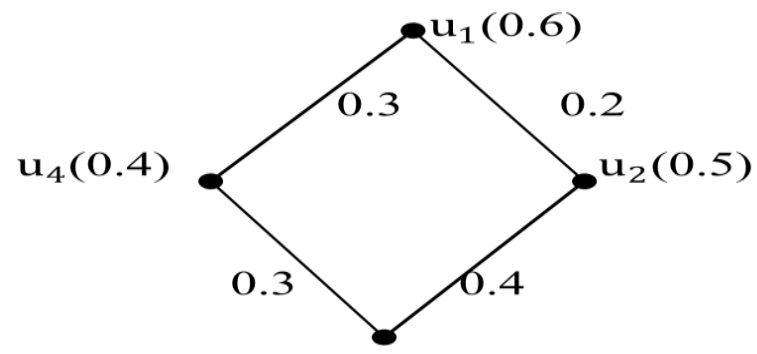

$$
u_{3}(0.7)
$$

Figure 5. 
" The fuzzy graph in fig.5 is neither regular nor complete regular fuzzy graph".

Remark 2.5. From the above examples, it is clear that in general there does not exist any relationship between regular fuzzy graphs and complete regular fuzzy graphs.

Theorem 2.6 ([7])."Let $\mathrm{G}:(\sigma, \mu)$ be a fuzzy graph on $\mathrm{G}^{*}:(\mathrm{V}, \mathrm{E})$ Then $\sigma$ is a constant function if and only if the following are equivalent :

(1). $\mathrm{G}$ is a regular fuzzy graph.

(2). G is a Complete regular fuzzy graph".

3. Properties of Complete Regular Fuzzy Graphs: Theorem 3.1."In any fuzzy graph $\mathrm{G}$, if $\sigma(\mathrm{v})>0$ for every vertex $\mathrm{v} \in \mathrm{V}$, thentd $(\mathrm{v})>0$, for every vertex $\mathrm{V} \in \mathrm{V}$.

Proof. Since $\sigma(v)>0$ for every vertex $\mathrm{v} \in \mathrm{V}$, $\mathrm{td}$ (v) > 0 , for every vertex $v \in \mathrm{V}$ "

Theorem 3.2."The maximum total degree of any vertex in a fuzzy graph with $\mathrm{p}$ vertices is $\mathrm{p}$.

Proof. For any vertex v,

$\operatorname{td}_{\mathrm{G}}(\mathrm{v})=\sum_{u v \in E} \mu(u v)+s(v)=\sum_{u v \in E} 1+1$

$=\mathrm{d}_{\mathrm{G}}{ }^{*}(\mathrm{v})+1=[\mathrm{p}-1]+1=\mathrm{p}$ "

Theorem 3.3."The total degree of a vertex $\mathrm{v}$ is $\sigma$ (v) if and only if the degree of $\mathrm{v}$ is 0 .

Proof. The total degree of a vertex $\mathrm{v}$ is $\operatorname{td}_{\mathrm{G}}(\mathrm{v})=\mathrm{s}(\mathrm{v})$

$\leftrightarrow \sum_{u v \in E} \mu(u v)+s(v) \leftrightarrow \sum_{u v \in E} \mu(u v)=0$

$\leftrightarrow \mathrm{d}_{\mathrm{G}}(\mathrm{v})=0$ ".

Corollary 3.4."The total degree of a vertex $\mathrm{v}$ is $\sigma$ (v) for every vertex $v$ in $G$ if and only if $G$ is a null fuzzy graph.

Proof.tdG (v) = s (v), for every vertex, $\mathrm{v} \in \mathrm{V}$ $\leftrightarrow \mathrm{dG} \quad(\mathrm{v})=0$, for every vertex

$v \in V \leftrightarrow G$ is a null fuzzy graph”.

Theorem 3.5."Every fuzzy graph is an induced fuzzy subgraph of a Complete regular fuzzy graph. Proof :Let $\mathrm{G}$ : $(\mathrm{V} ; \mathrm{E})$ be any fuzzy graph with $\mathrm{p}$ vertices and $\mathrm{q}$ edges.

If $\mathrm{G}$ is Complete regular, there is nothing to prove.

Suppose that $G$ is not Complete regular.

$$
\text { Let } \Delta \mathrm{t}=\max \{\operatorname{td}(\mathrm{v}) / \mathrm{v} \in \mathrm{V}\}
$$

Let us prove that $G$ is an induced fuzzy subgraph of a $\Delta \mathrm{t}$ - Complete regular Fuzzy Graph.

Take a copy G0 of G. Take any vertex $v$ with total degree less than $\Delta \mathrm{t}$

Join it $\mathrm{s}$ a copy $\mathrm{v} 0$ in G0

Assign $\min \{\sigma(\mathrm{v}), \Delta \mathrm{t}-\mathrm{tdG}(\mathrm{v})\}$ as the membership value of the edge vv0
Do this for all vertices with tdG0 (v) $<\Delta$ tinGo

Let the resultant fuzzy graph be G1.

For any vertex $\mathrm{v}$ with $\mathrm{td}(\mathrm{v})<\Delta \mathrm{t}$

If $\mu(\mathrm{vvo})=\min \{\sigma(\mathrm{v}), \Delta \mathrm{t}-\mathrm{tdG}(\mathrm{v})\}=\Delta \mathrm{t}-\mathrm{tdG}(\mathrm{v})$ Then

$\operatorname{tdG} 1(\mathrm{v})=\operatorname{tdG}(\mathrm{v})+\mu \quad(\mathrm{vvo})=\operatorname{tdG}(\mathrm{v})+\Delta \mathrm{t}-\operatorname{tdG}(\mathrm{v})=\Delta \mathrm{t}$

And all the vertices which have total degree $\Delta \mathrm{t}$ in $\mathrm{G}$ and their copies in G0 will have the same total degree $\Delta \mathrm{t}$ in $\mathrm{G} 1$. Also,

$\operatorname{tdG} 1(\mathrm{vo})=\operatorname{tdGo}(\mathrm{vo})+\mu \quad(\mathrm{vvo})=\operatorname{tdG}(\mathrm{v})+\mu \quad(\mathrm{vvo})=\Delta \mathrm{t}$

If this happens for every vertices with total degree less than $\Delta \mathrm{t}$ in $\mathrm{G}$ and their copies in $\mathrm{G} 0$, then the procedure stops here.

If for some vertex $\mathrm{v}$ with $\mathrm{td}(\mathrm{v})<\Delta \mathrm{t}$,

$\mu \quad(\mathrm{vvo})=\min \{\sigma \mathrm{G}(\mathrm{v}), \Delta \mathrm{t}-\mathrm{tdG}(\mathrm{v})\}=\sigma \mathrm{G}(\mathrm{v})$

Then $\operatorname{tdG} 1(\mathrm{v})<\Delta \mathrm{t}$ and $\operatorname{tdG} 1(\mathrm{v})<\Delta \mathrm{t}$

Now, repeat the above procedure for the fuzzy graph G1 and let the resultant fuzzy graph be $\mathrm{G}_{2}$. If all the vertices in $\mathrm{G}_{2}$ have total degree $\Delta \mathrm{t}$, stop.

Otherwise, continue the procedure till the vertices have total degree $\Delta t$ in the resultant fuzzy graph.

Let $\mathrm{n}=\left[\max \left\{\left(\frac{\Delta^{t}-t d_{G}(v)}{\sigma(v)} /{ }_{t d_{G}(v)}\right)<\Delta^{t}\right\}\right]$

Then the procedure stops after $\mathrm{n}$ steps with the $\Delta^{\wedge} \mathrm{t}-$ complete fuzzy graph $\mathrm{Gn}$.

Also $G$ is an induced fuzzy subgraph of $G n$. Here the number of vertices in

$$
\begin{aligned}
& \mathrm{G}_{\mathrm{n}}=\mathrm{p}+\mathrm{p}+2 \mathrm{p}+2^{2} \mathrm{p}+\ldots \ldots \ldots+2^{\mathrm{n}-1} \\
&==\mathrm{p}+\mathrm{p}\left(1+2+2^{2}+\ldots \ldots .2^{\mathrm{n}-1}\right) \\
&=\mathrm{p}+\left(\frac{2^{\mathrm{n}}-1}{2-1}\right) \mathrm{p} \\
&=\mathrm{p}+\left(2^{\mathrm{n}}-1\right) \mathrm{p}
\end{aligned}
$$$$
=2^{n} \mathrm{p}
$$

The number of edges in $\mathrm{G}_{\mathrm{n}}=\mathrm{nq}+\sum_{\text {wev }}\left\lfloor\frac{\Delta^{t}-t d_{G}(v)}{\sigma_{G(v)}}\right\rfloor$, 


\section{Complete Regular Fuzzy Graphs}
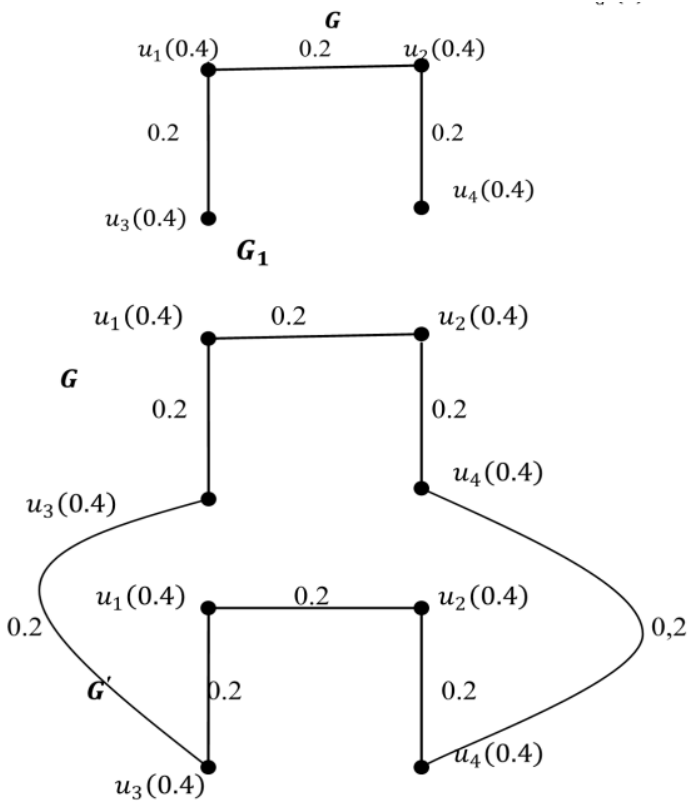

Figure 6. such that both $\sigma$ and $\mu$ are constant partially regular fuzzy graph. Then $G$ is a complete regular fuzzy graph if and only if $G$ is a partially regular fuzzy graph.

Proof : Assume that $\mathrm{G}$ is $\mathrm{K}$-complete regular fuzzy graph .

Let $\mu(\mathrm{uv})=\mathrm{c}$ for all $\mathrm{uv \epsilon V}$ and $\sigma(\mathrm{u})=\mathrm{c} 1$ for all $\mathrm{u} \in \mathrm{V}$ where $\mathrm{c}$ and $\mathrm{c} 1$ are constants.

Then td_G $(\mathrm{u})=\mathrm{dG}(\mathrm{u})+\sigma(\mathrm{u})$

$=\sum_{u \in v} \mu(u v)+\sigma(u)$

$\rightarrow \mathrm{k}=\mathrm{cd}^{\wedge} * \mathrm{G}(\mathrm{u})+\mathrm{c} 1$

$\rightarrow d^{*}{ }_{G}(\mathrm{u})=\frac{k-c_{1}}{c}$ for all $\mathrm{u} \in V$

So $G^{*}$ is regular abd hence $G$ is partially regular fuzzy graph.

Conversly assume that $G$ is a partially regular fuzzy graph

Let $\mathrm{G}^{*}$ be r-regular graph

Then

$$
\begin{aligned}
\operatorname{tdG}(\mathrm{u}) & =\mathrm{dG}(\mathrm{u})+\sigma(\mathrm{u}) \\
\rightarrow \operatorname{tdG}(\mathrm{u}) & =\mathrm{cd}^{*} \mathrm{G}(\mathrm{u})+\mathrm{c} 1
\end{aligned}
$$

all $\mathrm{u} \in \mathrm{V}$

$$
=\mathrm{cr}+\mathrm{c} 1 \text { for }
$$

So $\mathrm{G}$ is Complete regular fuzzy graph.

The following theorem will be helpful in studying various properties of complete regular fuzzy graph.

Theorem 3.7 : Let $\mathrm{G} 1:\left(\sigma_{1}, \mu_{1}\right)$ and $\mathrm{G}_{2}:\left(\sigma_{2}\right.$, $\mu_{2}$ ) be two fuzzy graphs such that $\sigma_{1}=\mu_{2}$ then $\sigma_{1}=\sigma_{2}$
Theorem 3.6."Let $\mathrm{G}:(\sigma, \mu)$ be a fuzzy graph

Proof :Since by the definition of a fuzzy graph, $\mu \_2(\mathrm{uv}) \leq \sigma \_2(\mathrm{u})^{\wedge} \sigma 2(\mathrm{v})$ for all $\mathrm{u}, \mathrm{v} \in \mathrm{V} 2$ we have $\min \mu_{2} \leq \sigma_{2}$

Now $\sigma_{1} \leq \mu_{2}$

$\rightarrow \sigma_{1} \leq \min \quad \mu_{2}$

Therefore $\quad \sigma_{1} \leq \min \quad \mu_{2} \leq \sigma_{2}$

$\rightarrow \sigma_{1} \leq \sigma_{2}$

4.Complete Regular Property of Union of Two Fuzzy Graphs

"Let $\mathrm{G}_{1}:\left(\sigma_{1}, \mu_{1}\right)$ and $\mathrm{G}_{2}:\left(\sigma_{2}, \mu_{2}\right)$ be any two fuzzy graphs underlying crisp Graphs

$\left(\mathrm{V}_{1}\right.$ and $\left.\mathrm{E}_{1}\right)$ and $\left(\mathrm{V}_{2}, \mathrm{E}_{2}\right)$ respectively.

i) If $u \in V_{1} \cup V_{2}$ and $u$ is arbitrary, then $t d_{\text {G1UG2 }}$ $=\left\{\begin{array}{l}t d_{G 1}(u), u \in V_{1} \\ t d_{G 2}(u), u \in V_{2}\end{array}\right\}$

ii) If $\mathrm{u} \in \mathrm{V}_{1} \cap V_{2}$ but no edge incident at $\mathrm{u}$ lies in $\mathrm{E}_{1} \cap E_{2}$. Then any edge incident at ' $\mathrm{u}$ ' is either in $E_{1}$ or $E_{2}$ but not both. Also all these edges will be included in $G_{1} \cup G_{2}$

$$
\underset{\wedge \sigma_{2}(u)}{t d_{G 1 \cup G 2}(u)}=t d_{G 1}(\mathrm{u})+t d_{G 2}(\mathrm{u})-\sigma_{1}(\mathrm{u})
$$

iii) If $u \in V_{1} \cap V_{2}$ but no edges incident at ' $u$ ' are in E1 $\mathrm{E} 2$ appear only once in G1U G2 andfor this uv

$$
\begin{gathered}
t d_{G 1 \cup G 2}(u)=t d_{G 1}(\mathrm{u})+t d_{G 2}(\mathrm{u})-\sigma_{1}(\mathrm{u}) \wedge \\
\sigma_{2}(u)-\sum_{u v E \mathrm{E} 1 \cap E 2} \mu_{1}(\mathrm{uv}) \wedge \mu_{2}(\mathrm{uv})
\end{gathered}
$$

Theorem 4.1: If G1 and G2 are two disjoint k-complete regular fuzzy graph, then $\mathrm{G}_{1} \cup G_{2}$ is k-complete regular graph.

Proof : Since G1 and G2 are disjoint fuzzy graphs

$$
\begin{array}{r}
t d_{G 1 \cup G 2}=\left\{\begin{array}{l}
t d_{G 1}(u), u \in V_{1} \\
t d_{G 2}(u), u \in V_{2}
\end{array}\right\} \\
=\mathrm{k} \text { for every } \mathrm{u} \in \mathrm{V}_{1} \cup V_{2}
\end{array}
$$

Therefore $\mathrm{G}_{1} \cup G_{2}$ is k-complete Graph" 


\section{REFERENCES}

[1] P.Bhattacharya, Some Remarks on Fuzzy Graphs, Pattern Recognition Letter, 6(1987), 297-302.

[2] John N.Modeson, Premch and S.Nair, Fuzzy Graphs and Fuzzy ypergraphs, Physica-verlag, Heidelberg, (2000).

[3] L.A.Zadeh, Fuzzy Sets, Inform. Control., 8(1965), 338-53.

[4] J.N.Mordeson and C.S.Peng, Operations on Fuzzy Graphs, Inform. Sci., 79(1994), 159-170.

[5] A.Nagoorgani and M.BasheerAhamed, Order and Size in Fuzzy Graph, Bulletin of Pure and Applied Sciences,22E(1)(2003), 145-148.

[6] A.Nagoorgani and K.Radha, Some Sequences in Fuzzy Graphs, Far East Journal of Applied Mathematics, 31(3)(2008),321-335.

[7] A.Nagoorgani and K.Radha, On Regular Fuzzy Graphs, Journal of Physical Sciences, 12(2008), 33-40.

[8] A.Nagoorgani and K.Radha, The Degree of a vertex in some fuzzy graphs, International Journal of Algorithms, Com-puting and Mathematics, 3(2009).

[9] A.Rosenfeld, Fuzzy Graphs, In: L. A. Zadeh, K.S. Fu, M. Shimura, Eds., Fuzzy sets and Their Applications, Academic Press, (1975), 77-95.

[10] K.Radha and M.Vijaya, The Total Degree of a vertex in some fuzzy graphs, Jamal Academic Research Journal :AnInterdisciplinary, special issue, (2014), 160-168.

[11] A.Nagoorgani and B.FathimaKani, Degree of a vertex in Alpha, Beta, Gamma Product of Fuzzy Graphs, Jamal Aca-demic Research Journal : An Interdisciplinary, special issue, (2014), 104-114.

[12] K.Radha and M.Vijaya, Totally Regular Property of Cartesian Product of two fuzzy graphs, Jamal Academic Research Journal : An Interdisciplinary, special issue, (2015), 647-652.

[13] K.Radha and M.Vijaya, Totally Regular Property of Composition of two fuzzy graphs", International journal of Pure and Applied Mathematical Sciences, 8(1)(2015), 87-100.

[14] K.Radha and M.Vijaya, Totally Regular Property of the join of two fuzzy graphs, International journal of Fuzzy Math-ematicalArchieve, $8(1)(2015), 9-17$.

[15] K.Radha and M.Vijaya, Totally Regular Property of Alpha Product of two fuzzy graphs, International Journal of Multi-disciplinary Research and Development, 3(4)(2016), 125-130.

[16] K.Radha and M.Vijaya, Totally Regular Property of Conjunction of two fuzzy graphs, Jamal Academic Research journal: An Interdisciplinary, special issue, (2016), 157-163.

[17] K.Radha and M.Vijaya, Regular and totally regular property of disjunction of two fuzzy graphs, International Journalof Multidisciplinary Research and Development, 4(4)(2017), 63-71. 